\title{
Uso de una checklist para reducir los errores cognitivos en la enseñanza práctica odontológica
}

\author{
Alejandro Iglesias-Linares, Rosa M. Yáñez-Vico, Enrique Solano-Reina
}

Introducción. Las estrategias de razonamiento heurístico predominan en el ámbito clínico. En odontología, y en particular en la práctica clínica diaria ortodóncica, se hace esencial diagnosticar bajo condiciones de presión, lo que fomenta los errores cognitivos por parte de los alumnos. El objetivo de este estudio fue proponer y validar una checklist como herramienta para disminuir los errores cognitivos diagnósticos por parte de los alumnos.

Sujetos y métodos. Treinta y seis estudiantes posgraduados realizaron dos evaluaciones clínicas de pacientes, una de la manera tradicional y otra aplicando la checklist propuesta.

Resultados. Se produjo una disminución estadísticamente significativa $(p<0,05)$ en los errores mediante la prueba de los rangos de Wilcoxon tras el uso de la checklist.

Conclusiones. La checklist propuesta ha demostrado su eficacia y ha sido evaluada positivamente por los alumnos; sin embargo, se debe prestar especial atención a los errores cognitivos de comisión porque aumenta de manera considerable su proporción.

Palabras clave. Checklist. Enseñanza sanitaria. Heurística. Recursos didácticos. Toma de decisiones clínicas.

Facultad de Odontología. Universidad de Sevilla. Sevilla, España.

Correspondencia: Prof. Alejandro Iglesias Linares. Facultad de Odontología. Universidad de Sevilla. Avicena, s/n. E-41009 Sevilla.

E-mail: aiglesiaslinares@us.es

Conflicto de intereses: No declarado.

Conflict of interests: None declared.

(c) 2013 FEM

\section{Use of checklist to reduce cognitive errors in dental practice teaching}

Introduction. Heuristic reasoning often dominate clinical judgments. Decision-making process in dentistry, and especially in orthodontics, is carry out under conditions of complexity and uncertainty. Therefore, the student decision processes are flawed by cognitive biases. The aim of our study was suggest and validate a checklist to reduce student diagnostic cognitive biases.

Subjects and methods. Thirty-six postgraduate students evaluated clinically patients without checklist and using the proposed checklist.

Results. Statistically significant difference $(p<0.05)$ was found using the Wilcoxon matched signed-rank test between two evaluations.

Conclusions. Effectiveness is prove by using the proposed checklist in reduce student cognitive errors and students have been positively evaluated it. Nevertheless, special attention is required to new increment of proportion of commission cognitive errors.

Key words. Checklist. Clinical decision making. Health education. Heuristic. Teaching resources.

\section{Introducción}

Las estrategias de razonamiento heurístico predominan en el ámbito clínico. Son procesos de pensamiento rápido e intuitivo muy frecuentes que se encuentran altamente influidos por numerosos factores, como las condiciones y el contexto ambiental, la edad y la experiencia [1]. En odontología, y en particular en la práctica clínica diaria ortodóncica, resulta esencial diagnosticar bajo condiciones de presión en un contexto acelerado, manejando mucha información y teniendo que tomar decisiones controvertidas [2]. En la enseñanza odontológica es muy importante la enseñanza práctica, que se fundamenta en el tratamiento real de pacientes por parte de los alumnos. Pero es muy frecuente que los alumnos se enfrenten a pacientes que han sido tratados por otros compañeros y ellos vean al paciente por primera vez con el tratamiento ortodóncico ya comenzado y tengan que continuar con el tratamiento. De la misma manera, también es muy frecuente que los alumnos no se acuerden de los pacientes o no relacionen los pacientes y el tratamiento que se está realizando. La toma de decisiones clínicas en este sentido, en 'primera línea' y bajo presión de tiempo, ocurre bajo una actividad cogni- 
Figura 1. Checklist propuesta a los alumnos posgraduados de ortodoncia.

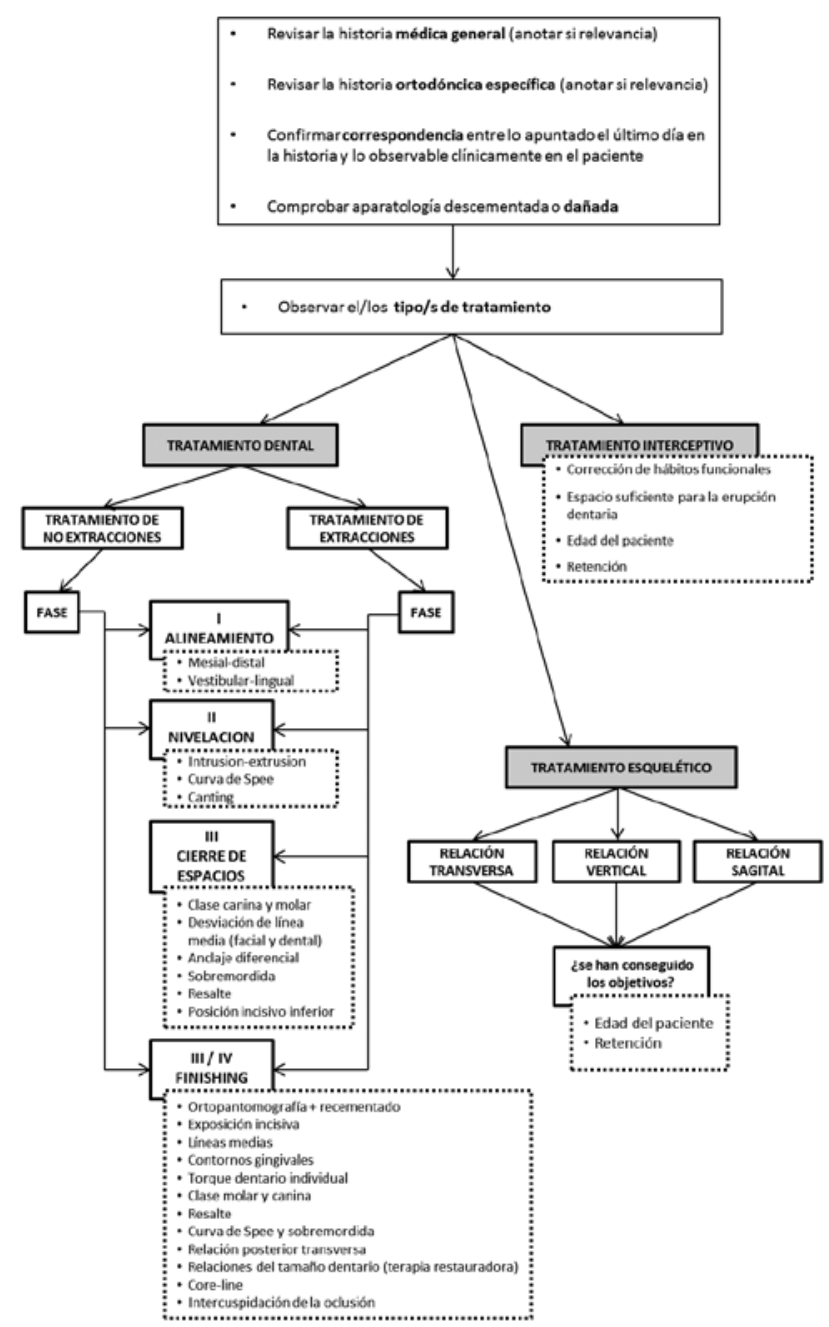

tiva extremadamente vulnerable al error. Esta realidad cognitiva obvia en gran medida los datos teóricos y normativos del sistema consciente, fomentando errores y fallos cognitivos inevitables por parte de los alumnos [3]. En este sentido, es importante encontrar soluciones reales a estos errores cognitivos de los cuales los odontólogos somos igualmente susceptibles y vulnerables, como las demás especialidades sanitarias [3].

Entre las soluciones que se han propuesto para solventar los problemas derivados de las imperfecciones y fallos de este sistema intuitivo destacan las prácticas reflexivas y el entrenamiento metacognitivo [4]. En este sentido, las checklists adquieren una especial relevancia por su éxito demostrado en medicina o en enfermería [5] para mejorar el razonamiento diagnóstico. Por ello, parece lógico sugerir que su uso ayude al aprendizaje para reducir los errores cognitivos en odontología, y en particular, en este caso, en la ortodoncia. El propósito de este estudio fue aplicar el concepto y uso de una checklist por parte de estudiantes de ortodoncia para la práctica clínica con pacientes como alternativa a la confianza de los alumnos en su memoria clínica, y estudiar la influencia de esta metodología en las competencias desarrolladas.

\section{Sujetos y métodos}

La cohorte de nuestro estudio fueron 36 licenciados de odontología que se encontraban cursando su especialidad posgraduada en ortodoncia. El programa posgraduado consta de 120 créditos ECTS que se realizan durante tres años de formación, siguiendo las directrices del programa Erasmus. Los estudiantes que participaron pertenecían al segundo $(n=18)$ y tercer $(n=18)$ año de formación. Para evaluar las competencias iniciales de las que los estudiantes partían, se realizó un cuestionario de evaluación abierto sobre el tratamiento de cinco pacientes, de tal manera que los estudiantes debían valorar cada uno de los cinco casos clínicos presentados y explicar de manera escrita, lo más exactamente posible, la secuencia de tratamiento que realizarían a cada paciente, así como cuestiones que quisieran preguntar o que consideraran de interés para la decisión del tratamiento. La cuantificación objetiva de estas competencias iniciales se realizó a través de la cuantificación de los errores cometidos en la secuencia de tratamiento expuesta por cada alumno, ya fueran errores de omisión o de comisión. A mayor puntuación obtenida, peores serían los resultados obtenidos.

Basándonos en el conocimiento de la especialidad por parte del equipo docente-investigador, así como en bibliografía especializada [6], desarrollamos una checklist para su uso en las prácticas posgraduadas de ortodoncia, con un formato doble: lista de elementos a verificar y secuencia iterativa de acciones (Fig. 1). Se explicaron a todos los alumnos el concepto y objetivos de las checklists, así como el modo de específico de utilización de la checklist propuesta. Los alumnos se familiarizaron así con la checklist propuesta para su aplicación en el aprendizaje práctico del tratamiento ortodóncico de los pacientes.

Finalmente, y tras dos meses de la evaluación inicial de los errores cognitivos por parte de los alum- 
nos, se realizó de nuevo la evaluación de sus competencias mediante la cumplimentación de un cuestionario abierto sobre el tratamiento ortodóncico de cinco pacientes. Los pacientes fueron los mismos, pero dispuestos en distinto orden. El transcurso de los dos meses entre una evaluación y otra aseguraba que los alumnos no pudieran recordar los casos de los pacientes ni la secuencia de tratamiento que propusieron en aquel momento.

Con el fin de conocer las percepciones y opiniones de los estudiantes tras el empleo de esta nueva metodología, se desarrolló un cuestionario cerrado de siete preguntas en el que los alumnos debían plasmar su acuerdo o desacuerdo respecto a los distintos ítems.

Se analizaron de modo univariado las variables cuantitativas (media \pm desviación estándar) de errores cometidos en la secuencia de tratamiento diagnosticada por los estudiantes, tanto sin el uso de la checklist como con su aplicación. Las diferencias entre las puntuaciones obtenidas con y sin el uso de la checklist, al ser dos muestras relacionadas, se compararon mediante análisis bivariado con el 95\% de significación, usando la prueba de los signos de Wilcoxon. La influencia de la variable 'año de formación' se analizó mediante la prueba $U$ de MannWhitney.

\section{Resultados}

La puntuación total de los promedios de errores que se producían en los estudiantes al decidir y explicar la secuencia de tratamiento resultó siempre mayor $(2,20-4,00)$ en la evaluación sin el uso de la checklist que cuando se utilizó la checklist como método coadyuvante $(0,20-1,40)$. Como se observa en la figura 2, estas puntuaciones se distribuían más ampliamente en la primera evaluación, mientras que tras la aplicación de la checklist, las puntuaciones obtenidas por los alumnos fueron más homogéneas. En torno al 30\% de los alumnos obtuvieron 0,8 de promedio y alrededor del $25 \%$ de los alumnos obtuvieron un promedio de 1,00.

La media de errores cometidos por los estudiantes en las secuencias de tratamiento propuestas en la primera evaluación, sin el uso de la checklist, oscilaba entre 2 y 3 , con un promedio de 2,89 (Tabla I). De la misma manera, en la tabla I pueden verse los resultados obtenidos tras la evaluación de los estudiantes aplicando la metodología de la checklist, lo que origina un menor número de errores (media en torno a 1; promedio: 0,9 ). Al evaluar comparativamente los errores cometidos por los estudiantes
Figura 2. Puntuaciones promedio obtenidas por porcentaje de alumnos.

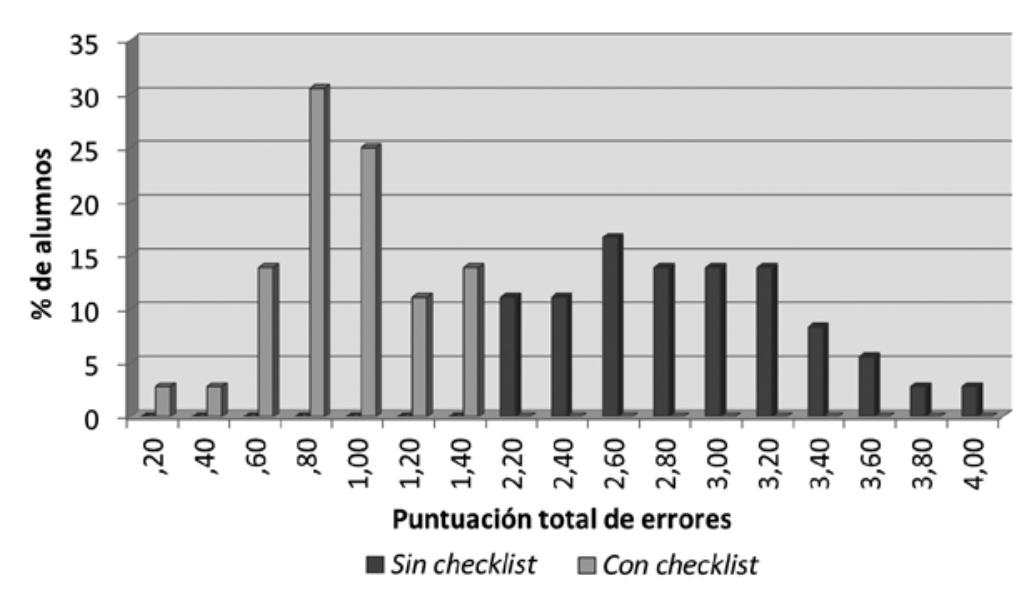

Tabla I. Comparación de errores cometidos por los estudiantes (media \pm desviación estándar) en la secuencia de tratamiento a realizar mediante la prueba de los signos de Wilcoxon.

\begin{tabular}{lcc}
\hline & Sin checklist & Con checklist \\
\hline Paciente 1 & $3,06 \pm 0,860$ & $1,19 \pm 0,920$ \\
\hline Paciente 2 & $2,97 \pm 1,000$ & $1,08 \pm 0,732$ \\
\hline Paciente 3 & $2,61 \pm 1,225$ & $1,08 \pm 0,732$ \\
\hline Paciente 4 & $2,78 \pm 1,017$ & $1,25 \pm 0,874$ \\
\hline Paciente 5 & $3,06 \pm 0,893$ & $1,06 \pm 0,924$ \\
\hline Promedio total & $2,8944 \pm 0,4720$ & $0,9222 \pm 0,2919$ \\
\hline
\end{tabular}

$p<0,05$ (estadísticamente significativo).

con y sin el uso de la checklist, los estudiantes cometieron menos errores cognitivos con el empleo de la checklist de manera estadísticamente significativa $(p<0,05)$.

En la tabla II se comparan los alumnos de segundo y tercer año de formación. Sin el uso de la checklist se encontraron diferencias estadísticamente significativas en los errores totales cometidos por los estudiantes, siendo mayor en los alumnos de segundo año de formación. Sin embargo, tras el uso de la checklist, no se hallaron diferencias significativas entre ambos grupos.

Tras las evaluaciones, los errores cognitivos producidos por los alumnos tras el diagnóstico y la ex- 
Tabla II. Comparación de errores cometidos entre los estudiantes de segundo y tercer curso en la secuencia de tratamiento a realizar mediante la prueba $U$ de Mann-Whitney.

\begin{tabular}{|c|c|c|c|c|}
\hline & \multicolumn{2}{|c|}{ Sin checklist } & \multicolumn{2}{|c|}{ Con checklist } \\
\hline & Segundo & Tercero & Segundo & Tercero \\
\hline Paciente 1 & $3,17 \pm 0,857$ & $2,94 \pm 0,873$ & $1,44 \pm 0,984$ & $0,94 \pm 0,802$ \\
\hline Paciente 2 & $3,28 \pm 0,958$ & $2,67 \pm 0,970$ & $1,06 \pm 0,802$ & $1,11 \pm 0,676$ \\
\hline Paciente 3 & $3,06 \pm 1,211^{a}$ & $2,17 \pm 1,098^{a}$ & $1,17 \pm 0,786$ & $1,00 \pm 0,686$ \\
\hline Paciente 4 & $3,11 \pm 1,023^{a}$ & $2,44 \pm 0,922^{a}$ & $1,33 \pm 0,970$ & $1,17 \pm 0,786$ \\
\hline Paciente 5 & $3,44 \pm 0,511^{a}$ & $2,67 \pm 1,029^{a}$ & $1,22 \pm 1,003$ & $0,89 \pm 0,832$ \\
\hline Promedio total & $3,2100 \pm 0,3900^{a}$ & $2,5800 \pm 0,3000^{a}$ & $1,0000 \pm 0,2800$ & $0,8400 \pm 0,2900$ \\
\hline
\end{tabular}

$p<0,05$. a Estadísticamente significativo.

Tabla III. Número y tipo de errores cometidos por los estudiantes de manera previa a la aplicación de la checklist $(n=521)$ y tras el uso de la checklist $(n=204)$

\begin{tabular}{|c|c|c|}
\hline & $\begin{array}{l}\text { Errores sin } \\
\text { checklist (\%) }\end{array}$ & $\begin{array}{l}\text { Errores con } \\
\text { checklist (\%) }\end{array}$ \\
\hline \multicolumn{3}{|l|}{ Errores de comisión } \\
\hline Añadir unidades de anclaje & 2 & 5 \\
\hline Ligar en continuo & 1 & 10 \\
\hline Recementar & 0 & 3 \\
\hline Elásticos intermaxilares & 4 & 2 \\
\hline Dobleces selectivas en el arco & 1 & 0 \\
\hline Cambio de arco & 7 & 20 \\
\hline \multicolumn{3}{|l|}{ Errores de omisión } \\
\hline Insuficiente anclaje & 10 & 5 \\
\hline No ligar en continuo & 9 & 4 \\
\hline No recementar & 7 & 10 \\
\hline No colocar elásticos intermaxilares & 2 & 8 \\
\hline No realizar dobleces selectivas en el arco & 13 & 11 \\
\hline No cambiar de arco & 1 & 1 \\
\hline Sobrecorrección & 3 & 1 \\
\hline \multicolumn{3}{|l|}{ Sesgos de sobreconfianza } \\
\hline No tener en cuenta la historia general del paciente & 12 & 3 \\
\hline No tener en cuenta la historia ortodóncica específica del paciente & 8 & 2 \\
\hline \multicolumn{3}{|l|}{ Errores por infraespecificación cognitiva } \\
\hline No preguntar datos necesarios & 10 & 3 \\
\hline \multicolumn{3}{|l|}{ Sesgos del efecto del orden } \\
\hline $\begin{array}{l}\text { Tener en cuenta casi exclusivamente datos iniciales, } \\
\text { sin considerar datos posteriores importantes }\end{array}$ & 1 & 5 \\
\hline $\begin{array}{l}\text { Tener en cuenta casi exclusivamente datos posteriores, } \\
\text { obviando datos iniciales importantes }\end{array}$ & 9 & 4 \\
\hline
\end{tabular}

posición de secuencia de tratamiento de los pacientes se agruparon principalmente en cinco clases (Tabla III):

- Errores de comisión.

- Errores de omisión.

- Sesgos de sobreconfianza.

- Errores por infraespecificación cognitiva.

- Sesgos del efecto del orden.

Estos tipos de errores no influyen de la misma manera en la decisión final del alumno. Cuando los alumnos no utilizaron la checklist, el mayor porcentaje de errores (45\%) se debía a errores de omisión, seguido de los sesgos producidos por la sobreconfianza (20\%). Sin embargo, al aplicar la metodología de la checklist, el mayor porcentaje de errores que se producían en los estudiantes eran errores de comisión (43\%), seguidos de los errores de omisión (40\%).

Los resultados obtenidos en la encuesta de opinión realizada a los alumnos muestran que la mayoría (76\%) habían encontrado fácil la resolución de los casos clínicos con la checklist, mientras que en la primera evaluación, sin la aplicación de ésta, únicamente el 32\% tenían la misma opinión (Tabla IV). En la tabla IV también se observan resultados favorables al uso de la checklist en cuanto a interés por parte del alumnado, ayuda y sensación de mejora. Sin embargo, la resolución de los casos clínicos continuaba resultando estresante al $49 \%$ de los estudiantes, aumentado esta cifra (80\%) sin el uso de la checklist.

\section{Discusión}

En las especialidades sanitarias, el objetivo final de la docencia es conseguir el aprendizaje por parte de los estudiantes en cuanto a adquisición de habilidades, resolución de problemas y construcción de patrones diagnósticos y terapéuticos que originen una correcta decisión diagnóstica y de tratamiento final. En el caso particular de la ortodoncia, constituye un tratamiento de larga duración, entre dos y dos años y medio, durante los cuales el paciente debe acudir a la clínica de manera mensual para continuar con su tratamiento. En estas visitas mensuales se 'diagnostica' la condición particular con la que acude el paciente para poder continuar de una $\mathrm{u}$ otra manera con el tratamiento. Por tanto, en cada visita del paciente, los alumnos deben diagnosticar y decidir la continuación de la secuencia de tratamiento, ya que ésta viene marcada en líneas muy generales en el diagnóstico y tratamiento iniciales, pero tiene numerosas variables que no se encuentran nunca mar- 
Tabla IV. Resultados de la encuesta de opinión realizada a los estudiantes.

\begin{tabular}{|c|c|c|c|c|c|c|}
\hline & & $\begin{array}{c}\text { Totalmente } \\
\text { de acuerdo } \\
(\%)\end{array}$ & $\begin{array}{c}\text { De } \\
\text { acuerdo } \\
(\%)\end{array}$ & $\begin{array}{l}\text { No sabe o } \\
\text { no contesta } \\
\text { (\%) }\end{array}$ & $\begin{array}{c}\text { En } \\
\text { desacuerdo } \\
\text { (\%) }\end{array}$ & $\begin{array}{c}\text { Totalmente } \\
\text { en desacuerdo } \\
(\%)\end{array}$ \\
\hline \multirow{2}{*}{$\begin{array}{l}\text { La resolución de los casos } \\
\text { clínicos me ha resultado fácil }\end{array}$} & Sin la checklist & 17 & 15 & 0 & 40 & 28 \\
\hline & Con la checklist & 32 & 44 & 0 & 16 & 9 \\
\hline \multirow{2}{*}{$\begin{array}{l}\text { La resolución de los casos clínicos } \\
\text { me ha resultado estresante }\end{array}$} & Sin la checklist & 39 & 41 & 0 & 14 & 6 \\
\hline & Con la checklist & 14 & 25 & 0 & 33 & 28 \\
\hline \multicolumn{2}{|c|}{ El uso de la checklist me resultó interesante tras su explicación } & 23 & 30 & 0 & 28 & 19 \\
\hline \multicolumn{2}{|c|}{$\begin{array}{l}\text { La aplicación de la checklist me ayudó en la resolución } \\
\text { de las secuencias de tratamiento }\end{array}$} & 35 & 46 & 0 & 16 & 3 \\
\hline \multicolumn{2}{|c|}{ Tengo sensación de mejora con el uso de la checklist } & 35 & 62 & 0 & 3 & 0 \\
\hline
\end{tabular}

cadas ni prefijadas, y que dependen de las condiciones particulares y específicas de cada paciente. De la misma manera ocurre en la práctica profesional.

El aprendizaje clínico ortodóncico posgraduado se fundamenta en la práctica con pacientes. Los alumnos deben tratar a pacientes que comienzan o incluso que ya están en tratamiento ortodóncico. A pesar de que reciben una enorme atención y tutorización por parte de sus profesores, desgraciadamente la ratio alumno/profesor no resulta suficiente para una completa monitorización. Es muy frecuente que los alumnos deban tomar decisiones menores ellos solos. Además, el factor tiempo obliga a realizar este tipo de prácticas clínicas bajo condiciones de presión, lo que condiciona frecuentes errores cognitivos. Otro hecho muy habitual es la falta de memoria clínica. Es muy común que los alumnos sean incapaces de recordar datos clínicos fundamentales de la historia de sus pacientes o el tipo de extracciones a realizar. De la misma manera, hemos podido comprobar que aunque el tutor les explique todos los procedimientos terapéuticos a realizar (haciendo especial énfasis en provisión de anclaje), resulta muy frecuente observar que al alumno 'se le olvida' algún detalle de los explicados y necesarios a realizar en el paciente, y no lo realiza, justificando este hecho la mayoría de las veces por la presión del tiempo y la falta de experiencia. En resumen, lo que estamos explicando son distintos errores cognitivos que se producen en el aprendizaje de ortodoncia, como sucede en otras especialidades sanitarias [3].
En odontología se han utilizado las checklists fundamentalmente para evaluar las habilidades del alumno $[7,8]$, aunque recientemente han demostrado el beneficio de su aplicación en la enseñanza de las habilidades clínicas en cirugía [9]. En cuanto a ortodoncia, sólo hemos encontrado checklists enfocadas al uso por parte de los profesionales de la ortodoncia y en el campo específico de la finalización óptima del tratamiento del paciente ortodóncico [10-12]. Así, únicamente son de aplicación para el acabado último de los casos, esto es, para dar el alta al paciente tras unos dos años de tratamiento. Por tanto, no son aplicables durante las distintas sesiones de tratamiento ortodóncico, como la que se propone en este artículo, ya que los ítems difieren notablemente al buscar distintos objetivos. Aun así, es importante destacar la utilidad encontrada por parte de los profesionales, ya que su aplicación optimiza en gran medida el tiempo en la clínica [1012]. Se incide en que su empleo puede ayudar como criterio de calidad en la finalización de un tratamiento y ser de utilidad como documentación médico-legal [11]. La unión de estos datos, junto con los excelentes resultados obtenidos en otros campos sanitarios [6,7], hace tomar especial relevancia a la evaluación de las checklists para disminuir los errores cognitivos en ortodoncia y a su utilización para implementar el aprendizaje clínico por parte de alumnos posgraduados que se encuentran cursando la especialidad de ortodoncia.

Por ello, desarrollamos una checklist de uso específico ortodóncico con la finalidad de que sirviera 
de ayuda a los estudiantes de ortodoncia cuando tuvieran que decidir las secuencias de tratamiento de sus pacientes y las distintas partes de su ejecución. Hemos podido comprobar que la aplicación de esta metodología, después de haberse familiarizado los estudiantes con ella, originó una disminución estadísticamente significativa $(p<0.05)$ de los errores por parte de los alumnos en la secuencia de tratamiento decidida por ellos tras el uso de la checklist propuesta, en comparación con su no uso. La aplicación de esta metodología ha sido especialmente positiva para los alumnos del segundo año de formación porque, tras el uso de la checklist, ambos grupos en distinto año de formación se han equiparado en el número de errores cometidos.

Es igualmente interesante el estudio de los errores que se han producido, ya que éstos resultaron ser distintos, o por lo menos distribuirse de distinto modo, con y sin checklist. Con el uso de la checklist, los errores típicamente cognitivos, sesgos heurísticos como los errores por infraespecificación cognitiva y los sesgos de sobreconfianza, disminuyeron drásticamente. Sin embargo, se produjo un aumento en la proporción de errores de comisión (adviértase que nos estamos refiriendo a porcentajes del total de errores, que son muy diferentes en ambas evaluaciones, por tanto no quiere decir que el número toral de errores de comisión aumente con el uso de la checklist). Una posible explicación a este hecho resida en que el alumno, al ver en la checklist la posibilidad de realizar determinados procedimientos y no encontrarse seguro teóricamente ni reforzado por su aprendizaje teórico, crea que existe 'esa necesidad', ya que ha identificado el problema. En este punto, por tanto, debemos incidir más en los estudiantes en cuanto a la secuenciación correcta, en cuanto a orden de priorización de los procedimientos, ya que no se trataba tanto de procedimientos no indicados, sino de momento de tratamiento no indicado y, por tanto, erróneo.

En cuanto a la opinión de los estudiantes al evaluar los casos clínicos para decidir el tratamiento a ejecutar y su secuencia se obtuvieron resultados favorables en cuanto a grado de dificultad, sensación de mejora, interés y utilidad. Un dato interesante es que la mayoría de los estudiantes $(80 \%)$ encontra- ron el procedimiento estresante, lo que viene a apoyar las condiciones heurísticas de su aprendizaje. Aun usando una estrategia específica para este tipo de procesamiento heurístico [6], aproximadamente la mitad de los alumnos continuaban hallando el procedimiento estresante.

En conclusión, los resultados obtenidos sugieren que la aplicación de una checklist específica para el tratamiento ortodóncico ayuda a disminuir de manera significativa los errores cognitivos por parte de los estudiantes de ortodoncia. El procedimiento, además, es bien aceptado por los alumnos. Sin embargo, se debe incidir más teóricamente en la priorización de las acciones a realizar, ya que los estudiantes son más conscientes de determinados procedimientos al comprobarlos en la checklist, por lo que cuando cometen algún error aun aplicando la checklist, en un alto porcentaje son errores de comisión.

Bibliografía

1. Croskerry P. A universal model for diagnostic reasoning. Acad Med 2009; 84: 1022-8.

2. Hicks EP, Kluemper GT. Heuristic reasoning and cognitive biases: are they hindrances to judgments and decision making in orthodontics? Am J Orthod Dentofacial Orthop 2011; 139: 297-304.

3. Croskerry P. The importance of cognitive errors in diagnosis and strategies to minimize them. Acad Med 2003; 78: 775-80.

4. Mamede S, Schimdt HG, Rikers G. Diagnostic errors and reflective practice in medicine. J Eval Clin Pract 2007; 13: 138-45.

5. Gawande A. The checklist manifesto. How to get things right. New York: Metropolitan Books; 2009.

6. Ely J, Graber M, Croskerry P. Checklist to reduce diagnostic errors. Acad Med 2011; 86: 307-13.

7. Macluskey M, Durham J, Balmer C, Bell A, Cowpe J, Dawson L, et al. Dental student suturing skills: a multicentre trial of a checklist-based assessment. Eur J Dent Educ 2011; 15: 244-9.

8. Durham J, Balmer C, Bell A, Cowan G, Cowpe J, Crean SJ, et al. A generic consensus assessment of undergraduate competence in forceps exodontia in the United Kingdom. Eur J Dent Educ 2010; 14: 210-4.

9. Macluskey M, Hanson C. The retention of suturing skills in dental undergraduates. Eur J Dent Educ 2011; 15: 42-6.

10. Poling R. A method of finishing the occlusion. Am J Orthod Dentofacial Orthop 1999; 115: 476-87.

11. Kortam SI, El-Beialy AR, Mostafa YA. Retention checklist. J Clin Orthod 2007; 41: 382-3.

12. El-Beialy AR, Kortam SI, Mostafa YA. Case finishing checklist. J Clin Orthod 2005; 39: 322-3. 